\title{
Hochschild Homology Groups of System Quiver Algebras of Maximal Tame Type
}

\author{
Chunling He*, Huiru Chen, Zhibing Liu \\ College of Mathematics and Physics, Huanggang Normal University, Huanggang 438000, China. \\ * Corresponding author. Tel.: 15271619026; email: 59103008@qq.com \\ Manuscript submitted April 7, 2017; accepted September 12, 2017. \\ doi: 10.17706/ijapm.2017.7.4.259-267
}

\begin{abstract}
Let $\Lambda_{\mathrm{i}}=k Q / I_{i}$ be system quiver algebras of maximal tame representation types. In this paper, based on the minimal projective bimodule resolutions, we calculate explicitly the dimensions of all Hochschild homology groups of the system quiver of maximal tame representation types.
\end{abstract}

Key words: System quiver algebra, aximal tame representation type, minimal projective bimodule resolution, hochschild cohomology group.

\section{Introduction}

The differential equation $\left\{\begin{array}{l}\frac{d(x(t))}{d t}=F x(t)+G u(t) \\ y(t)=H x(t)\end{array}\right.$, Provides the linear time invariant dynamical system $\sum=(G ; F ; H)$, where $x(t) \in k^{n}, u(t) \in k^{m}, y(t) \in k^{p}$ are vector variables, and $G ; F ; H$ are $n \times m ; n \times n ; p \times n$ matrix respectively. Hazewinkel first obtained the one to one correspondence of every linear time invariant dynamical system $\sum=(G ; F ; H)$ and the dimensional vector $(m ; n ; p)$ of the following system quiver $Q$ in [1].

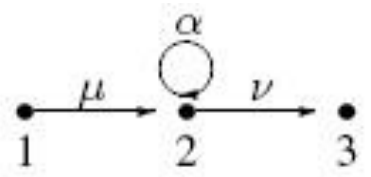

The equivalence classes of a dynamical system are one-to-one correspondence with the isomorphism classes of the finite dimensional representations of the system quiver. Elements of the repreesentation theory had been explained in [2]. Longcai Li et al. classfied all the system quiver algebras according their representation type,and found the list of system quiver algebras of maximal tame representation type [3]. System quiver algebra of minimal wild type had been discussed in [4].

Let $\Lambda$ is a system quiver algebra. In the isomorphism and dual sense, $\Lambda$ is tame type if and only if $\Lambda$ is (or degenerate) $k Q / I$, where $I$ is correspond to $T$. The algebra is called the system quiver algebra of maximal tame type. 
$T$

(1) $I_{1}=\left(\alpha^{2}\right)$;

(2) $I_{2}=\left(\alpha^{3}, \mu v, \mu \alpha v\right)$;

(3) $I_{3}=\left(\mu v, \alpha^{2} v, \mu \alpha^{2}, \alpha^{3}\right)$;

(4) $I_{4}=\left(\mu \alpha, \alpha^{2} v, \alpha^{4}\right)$;

(5) $I_{5}=\left(\alpha v, \mu \alpha, \alpha^{m}\right),(m \geq 5)$.

Hochschild homology group was introduced by Hochschild in 1945 in [5]. Let $\Lambda$ be the finite dimensional combination basic algebra (Including identical element 1) in number field K. Its enveloping algebra was defined as $\Lambda^{e}=\Lambda^{o p} \otimes_{k} \Lambda, \Lambda^{o p}$ be the reflexive algebra of $\Lambda . M$ be the finite dimensional $\Lambda-\Lambda-$ dual-mode in number field K, then coefficients in the $M$ of algebraic $\Lambda$, which Homology group at the n-th order is defined as $H_{n}(\Lambda, M)=\operatorname{Tor}_{n}^{\Lambda^{e}}(M, \Lambda)$

Especially, when $M=\Lambda$, then say $H H_{n}(\Lambda)=H H_{n}(\Lambda, \Lambda)$, known as the n-th order algebraic Hochschild homology group.

The arrow diagram and its development and application, provided a effective method for calculating the Hochschild homology of algebra group.

Bardzell gave the explicit description of construction of minimal projective bimodule resolutions of monomial algebras with directed paths. Using Bardzell's method, the minimal projective bimodule resolutions of the system quiver algebras of maximal tame representation type is constructed in [6]. In this thesis, based on the resolutions, we calculate explicitly the dimensions of all Hochschild homology groups of the system quiver algebras of maximal tame representation type by means of combinatorics. Thus we get a further understanding of the homology property of those algebras.

\section{Minimal Projective Bimodule Resolution}

In the section, we first introduce the minimal projective bimodule resolutions of the system quiver algebras of maximal tame representation type constructed by Dandan Zhang in [7].

By the correspondence of the list in introduction, it can obtain the following ideals corresponding to the system quiver algebras of maximal tame type $\Lambda_{i}=k Q / I_{i}: I_{1}=\left(\alpha^{2}\right), I_{2}=\left(\alpha^{3}, \mu v, \mu \alpha v\right)$, $I_{3}=\left(\mu v, \alpha^{2} v, \mu \alpha^{2}, \alpha^{3}\right), I_{4}=\left(\mu \alpha, \alpha^{2} v, \alpha^{4}\right), I_{5}=\left(\alpha v, \mu \alpha, \alpha^{m}\right), \quad(m \geq 5)$.

Let $B_{i}$ be a series of $k$ - bases of $\Lambda_{i}$, and $e_{1}, e_{2}, e_{3}$ be the primitive idempotent element corresponding to the vertexes $1,2,3$ respectively, we have

$$
\begin{gathered}
B_{1}=\left\{e_{1}, e_{2}, e_{3}, \mu, \alpha, v, \mu v, \alpha v, \mu \alpha, \mu \alpha v\right\}, \operatorname{dim}_{k} \Lambda_{1}=10 \\
B_{2}=\left\{e_{1}, e_{2}, e_{3}, \mu, \alpha, v, \mu \alpha, \alpha v, \alpha^{2}, \mu \alpha^{2}, \alpha^{2} v, \mu \alpha^{2} v\right\}, \operatorname{dim}_{k} \Lambda_{12}=12 \\
B_{3}=\left\{e_{1}, e_{2}, e_{3}, \mu, \alpha, v, \mu \alpha, \alpha v, \alpha^{2}, \mu \alpha v\right\}, \operatorname{dim}_{k} \Lambda_{3}=10 ; \\
B_{4}=\left\{e_{1}, e_{2}, e_{3}, \mu, \alpha, v, \mu v, \alpha v, \alpha^{2}, \alpha^{3}\right\}, \operatorname{dim}_{k} \Lambda_{4}=10
\end{gathered}
$$




$$
B_{5}=\left\{e_{1}, e_{2}, e_{3}, \mu, \alpha, v, \mu v, \alpha^{2}, \alpha^{3}, \cdots, \alpha^{m-1}\right\}, \operatorname{dim}_{k} \Lambda_{5}=m+5
$$

And then, we construct the minimal projective bimodule resolutions of $\Lambda_{i}$. Let $\Lambda_{i}^{e}=\Lambda_{i}^{o p} \otimes_{k} \Lambda_{i}$ be the enveloping algebra of $\Lambda_{i}$, writed for

$$
\begin{aligned}
& \otimes:=\otimes_{k} . P_{n}^{i}:=\underset{p \in A P_{i}(n)}{\amalg} \Lambda_{i} o(p) \otimes t(p) \Lambda_{i}, \text { where the constructions of } A P_{i}(n) \text { are as follows: } \\
& A P_{i}(0)=\left\{e_{1}, e_{2}, e_{3}\right\}, \quad A P_{i}(1)=\{\mu, \alpha, v\} . \\
& \text { For } \Lambda_{1}, A P_{1}(n)=\left\{\alpha^{n}\right\}, \text { When } n \geq 2 . \\
& \text { For } \Lambda_{2}, A P_{2}(2)=\left\{\alpha^{3}, \mu v, \mu \alpha v\right\} . \text { When } n \geq 3,
\end{aligned}
$$

$$
A P_{2}(n)\left\{\begin{array}{l}
\left\{\alpha^{\frac{3 n-1}{2}}\right\} \\
\left\{\alpha^{\frac{3 n}{2}}\right\} \text { when } n \text { is an odd number; } \\
\text { when } n \text { is an even number. }
\end{array}\right.
$$

For $\Lambda_{3}, A P_{3}(2)=\left\{\mu v, \alpha^{2} v, \mu \alpha^{2}, \alpha^{3}\right\}$. When $n \geq 3$,

$$
A P_{3}(n)=\left\{\begin{array}{l}
\left\{\mu \alpha^{\frac{3 n-5}{2}} v, \mu \alpha^{\frac{3 n-3}{2}}, \alpha^{\frac{3 n-3}{2}} v, \alpha^{\frac{3 n-1}{2}}\right\} \\
\left\{\mu \alpha^{\frac{3 n-4}{2}} v, \mu \alpha^{\frac{3 n-2}{2}}, \alpha^{\frac{3 n-3}{2}} v, \alpha^{\frac{3 n}{2}}\right\} \\
\text { when } \mathrm{n} \text { is an even number. }
\end{array}\right.
$$

For $\Lambda_{4}, A P_{4}(2)=\left\{\mu \alpha, \alpha^{2} v, \alpha^{4}\right\}$. When $n \geq 3$

$$
A P_{4}(n)=\left\{\begin{array}{r}
\left\{\mu \alpha^{2 n-2}, \mu \alpha^{2 n-4} v, \alpha^{2 n-1}, \alpha^{2 n-2} v\right\} \\
\text { when } \mathrm{n} \text { is an odd number; } \\
\left\{\mu \alpha^{2 n-3}, \mu \alpha^{2 n-4} v, \alpha^{2 n}, \alpha^{2 n-2} v\right\} \\
\text { when } \mathrm{n} \text { is an even number. }
\end{array}\right.
$$

For $\Lambda_{5}, A P_{5}(2)=\left\{\mu \alpha, \alpha v, \alpha^{m}\right\},(m \geq 5)$. When $n \geq 3$,

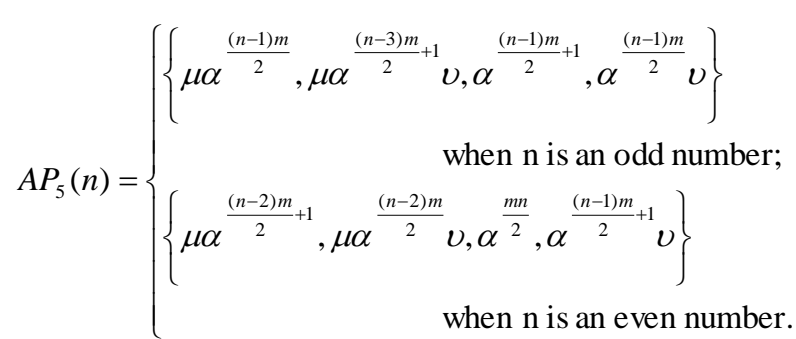


For all $p^{n} \in A P_{i}(n)$, define $\sup \left(p^{n}\right)=\left\{p^{n-1} \in A P_{i}(n-1): p^{n-1}\right.$ is a subpath of $\left.p^{n}\right\}$

Let $\mathrm{n}$ be an odd number, $p^{n} \in A P_{i}(n)$, then $\sup \left(p^{n}\right)=\left\{p_{1}^{n-1}, p_{2}^{n-1}\right\}, \quad$ where $p_{l}^{n-1} \in A P_{i}(n-1)$, $(l=1,2)$, and there exists only one subpath $R_{2}^{1}$ and $L_{1}^{2}$, such that $p^{n}$ exists the following decomposition: $p^{n}=p_{1}^{n-1} R_{2}^{1}=L_{1}^{2} p_{2}^{n-1}$, and then $p^{n+1} \in A P_{i}(n+1), \sup \left(p^{n+1}\right)=\left\{p_{1}^{n}, p_{2}^{n}, \cdots, p_{s}^{n}\right\}, \quad(i=1, \cdots, s)$, where $p^{n+1} \in \theta_{i} p_{i}^{n} \mu_{i}, \theta_{i}$ and $\mu_{i}$ are the supplemental subpaths of $p_{i}^{n}$ with respect to $p^{n+1}$. Define $\delta_{n}^{i}: P_{n}^{i} \rightarrow P_{n-1}^{i}$ the following form:

$$
\begin{gathered}
\delta_{n}^{i}\left(o\left(p^{n}\right) \otimes t\left(p^{n}\right)\right)=L_{1}^{2} \otimes t\left(p^{n}\right)-o\left(p^{n}\right) \otimes R_{2}^{1} \\
\delta_{n+1}^{i}\left(o\left(p^{n+1}\right) \otimes t\left(p^{n+1}\right)\right)=\sum_{i=1}^{s} \theta_{i} \otimes \mu_{i}
\end{gathered}
$$

And then it follows the following theorem according to [6].

Theorem 1 For the above the system quiver algebras $\Lambda_{i}$ of maximal tame representation type, the complex

$$
\left(P^{i}, \delta^{i}\right) \cdots \rightarrow P_{n+1}^{i} \stackrel{\delta_{n+1}^{i}}{\rightarrow} P_{n}^{i} \stackrel{\delta_{n}^{i}}{\rightarrow} \cdots \stackrel{\delta_{3}^{i}}{\rightarrow} P_{2}^{i} \stackrel{\delta_{2}^{i}}{\rightarrow} P_{1}^{i} \stackrel{\delta_{1}^{i}}{\rightarrow} P_{0}^{i} \rightarrow 0
$$

is the minimal projective $\Lambda_{\mathrm{i}}^{e}-$ resolution of $\Lambda_{i}$.

\section{Hochschild Homology Group}

Let $X, Y$ is a set that consists of all consistent elements of $k Q$, and $X \odot Y=\{(x, y) \in X \times Y$ $\mid t(x)=o(y), \quad t(y)=o(x)\}$.Then $k(X \odot Y)$ is a linear space whose base is $X \odot Y$. In the section, we calculate the dimensions of all Hochschild homology groups of the system quiver algebras of maximal tame representation type by means of combinatorics.

Acting on the minimal projection $\Lambda_{\mathrm{i}}^{e}-$ resolution $\left(P^{i}, \delta^{i}\right)$ of $\Lambda_{i}$ by the functor $-\otimes_{\Lambda_{i}^{e}} \Lambda_{i}$, it follows $\cdots \rightarrow P_{n}^{i} \otimes_{\Lambda_{i}^{e}} \Lambda_{i} \stackrel{\delta_{n}^{i} \otimes 1}{\rightarrow} P_{n+1}^{i} \otimes_{\Lambda_{i}^{e}} \Lambda_{i} \rightarrow \cdots P_{1}^{i} \otimes_{\Lambda e_{i}} \Lambda_{i} \stackrel{\delta_{1}^{i} \otimes 1}{\rightarrow} P_{0}^{i} \otimes_{\Lambda_{i}^{e}} \Lambda_{i} \rightarrow 0$

Note that

$$
\begin{gathered}
P_{n}^{i} \otimes_{\Lambda_{i}^{e}} \Lambda_{i} \cong \coprod_{p \in A P_{i}(n)}(o(p) \otimes t(p)) \otimes_{k Q_{0}} \Lambda^{i} \cong \coprod_{p \in A P_{i}(n)}(o(p) \otimes t(p)) \otimes_{k Q_{0}}\left(\oplus_{k, l=1}^{3} e_{k} \Lambda^{i} e_{l}\right) \\
\cong k\left(B_{i} \otimes A P_{i}(n)\right) .
\end{gathered}
$$

It follows Bardzell chain complex

$$
\cdots \rightarrow k\left(B_{i} \odot A P_{i}(n)\right) \stackrel{\tau_{n}^{i}}{\rightarrow} k\left(B_{i} \odot A P_{i}(n-1)\right) \cdots
$$




$$
\rightarrow k\left(B_{i} \odot A P_{i}(1)\right) \stackrel{\tau_{1}^{i}}{\rightarrow} k\left(B_{i} \odot A P_{i}(0) \rightarrow 0\right.
$$

where, for all $\left(c, p^{n}\right) \in\left(B_{i} \odot A P_{i}(n)\right)$, if $n$ is an odd number, then

$$
\tau_{n}^{i}\left(c, p^{n}\right)=\left(c \lambda, p_{1}^{n-1}\right)-\left(\theta c, p_{2}^{n-1}\right)
$$

where $p^{n}=\lambda p_{1}^{n-1}=p_{2}^{n-1} \theta, p_{1}^{n-1}, p_{2}^{n-1} \in A P_{i}(n-1)$ aresubpaths of pn. And if is an even number, then

$$
\tau_{n}^{i}\left(c, p^{n}\right)=\sum_{k}\left(\theta_{k} c \lambda_{k}, p^{n-1}\right)
$$

where $p_{k}^{n-1}$ is any one of $\sup \left(p^{n}\right)$ such that $p^{n}=\lambda_{k} p_{k}^{n-1} \theta_{k}$.

Theorem 2 Let $\Lambda_{1}=k Q / I_{1}=k Q /\left(\alpha^{2}\right)$, then

$$
\left|B_{1} \odot \mathrm{A} P_{1}(n)\right|= \begin{cases}4 & \text { when } n=0 ; \\ 2 & \text { when } n \geq 1\end{cases}
$$

and

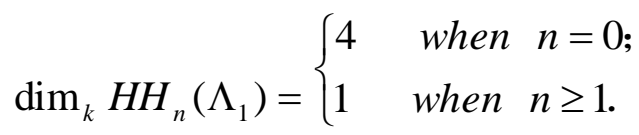

Let $\Lambda_{2}=k Q / I_{2}=k Q /\left(\alpha^{3}, \mu v, \mu \alpha v\right)$, then

$$
\left|B_{2} \odot \mathrm{A} P_{2}(n)\right|= \begin{cases}5 & \text { when } n=0 \\ 3 & \text { when } n \geq 1\end{cases}
$$

and

$$
\begin{aligned}
& \operatorname{dim}_{k} H H_{n}\left(\Lambda_{2}\right)= \begin{cases}5 & \text { when } n=0 ; \\
2 & \text { when } n \geq 1 .\end{cases} \\
& \text { Let } \Lambda_{3}=k Q / I_{3}=k Q /\left(\mu v, \alpha^{2} v, \mu \alpha^{2}, \alpha^{3}\right) \text {, then } \\
& \left|B_{3} \odot \mathrm{A} P_{3}(n)\right|= \begin{cases}5 & \text { when } n=0 ; \\
3 & \text { when } n \geq 1 .\end{cases}
\end{aligned}
$$

and 


$$
\begin{aligned}
& \qquad \operatorname{dim}_{k} H H_{n}\left(\Lambda_{3}\right)= \begin{cases}5 & \text { when } n=0 ; \\
2 & \text { when } n \geq 1 .\end{cases} \\
& \text { Let } \Lambda_{4}=k Q / I_{4}=k Q /\left(\mu v, \alpha^{2} v, \alpha^{4}\right) \text {, then } \\
& \qquad\left|B_{4} \odot \mathrm{AP}_{4}(n)\right|= \begin{cases}6 & \text { when } n=0 ; \\
4 & \text { when } n \geq 1 .\end{cases}
\end{aligned}
$$

and

$$
\operatorname{dim}_{k} H H_{n}\left(\Lambda_{4}\right)= \begin{cases}6 & \text { when } n=0 ; \\ 3 & \text { when } n \geq 1\end{cases}
$$

Let $\Lambda_{5}=k Q / I_{5}=k Q /\left(\alpha v, \mu \alpha, \alpha^{m}\right),(m \geq 5)$, then

$$
\begin{aligned}
& \left|B_{5} \odot \mathrm{A} P_{5}(n)\right|= \begin{cases}m+2 & \text { when } n=0 \\
m & \text { when } n \geq 1\end{cases} \\
& \operatorname{dim}_{k} H H_{n}\left(\Lambda_{5}\right)=\left\{\begin{array}{l}
m+2 \quad \text { when } n=0 ; \\
m-1 \quad \text { when } n \geq 1
\end{array}\right.
\end{aligned}
$$

Proof: Here, the conclusion of $\Lambda_{3}$ is only proved and the others can similarly be proved. From the second section, it follows

$$
B_{3}=\left\{e_{1}, e_{2}, e_{3}, \mu, \alpha, v, \mu \alpha, \alpha v, \alpha^{2}, \mu \alpha v\right\}, \operatorname{dim}_{k} \Lambda_{3}=10, A P_{3}(0)=\left\{e_{1}, e_{2}, e_{3}\right\}, A P_{3}(1)=\{\mu, \alpha, v\}
$$

For $\Lambda_{3}, A P_{3}(2)=\left\{\mu v, \alpha^{2} v, \mu \alpha^{2}, \alpha^{3}\right\}$, when $n \geq 3$, and $\mathrm{n}$ is an odd number,

$$
A P_{3}(n)=\left\{\mu \alpha^{\frac{3 n-5}{2}} v, \mu \alpha^{\frac{3 n-3}{2}}, \alpha^{\frac{3 n-3}{2}} v, \alpha^{\frac{3 n-1}{2}}\right\}
$$

when $n \geq 3$, and $n$ is an even number,

$$
A P_{3}(n)=\left\{\mu \alpha^{\frac{3 n-4}{2}} v, \mu \alpha^{\frac{3 n-2}{2}}, \alpha^{\frac{3 n-2}{2}} v, \alpha^{\frac{3 n}{2}}\right\}
$$

so

$$
B_{3} \odot A P_{3}(0)=\left\{\left(e_{1}, e_{1}\right),\left(e_{2}, e_{2}\right),\left(\alpha, e_{2}\right),\left(\alpha^{2}, e_{2}\right)\left(e_{3}, e_{3}\right)\right\},
$$




$$
\begin{gathered}
B_{3} \odot A P_{3}(1)=\left\{\left(e_{2}, \alpha\right),(\alpha, \alpha),\left(\alpha^{2}, \alpha\right)\right\}, \\
B_{3} \odot A P_{3}(2)=\left\{\left(e_{2}, \alpha^{3}\right),\left(\alpha, \alpha^{3}\right),\left(\alpha^{2}, \alpha^{3}\right)\right\},
\end{gathered}
$$

when $n \geq 3$, and $\mathrm{n}$ is an odd number,

$$
B_{3} \odot P_{3}(n)=\left\{\left(e_{2}, \alpha^{\frac{3 n-1}{2}}\right),\left(\alpha, \alpha^{\frac{3 n-1}{2}}\right),\left(\alpha^{2}, \alpha^{\frac{3 n-1}{2}}\right)\right\},
$$

when $n \geq 3$, and $\mathrm{n}$ is an even number,

$$
B_{3} \odot P_{3}(n)=\left\{\left(e_{2}, \alpha^{\frac{3 n}{2}}\right),\left(\alpha, \alpha^{\frac{3 n}{2}}\right),\left(\alpha^{2}, \alpha^{\frac{3 n}{2}}\right)\right\} .
$$

so

$$
\left|B_{3} \odot \mathrm{A} P_{3}(n)\right|=\left\{\begin{array}{lll}
5 & \text { when } & n=0 \\
3 & \text { when } & n \geq 1
\end{array}\right.
$$

For proving the next conclusion, we can sequence the base of ${ }^{B_{3}} \odot A P_{3}(n)$ in accordance with the order in the previous proving, and still denote its corresponding matrix by $\tau_{n}^{3}$.

From (1) and (2), it follows:

$$
\begin{gathered}
\tau_{1}^{3}\left(e_{2}, \alpha\right)=\left(e_{2} \alpha, e_{2}\right)-\left(\alpha e_{2}, e_{2}\right)=0, \\
\tau_{1}^{3}(\alpha, \alpha)=\left(\alpha^{2}, e_{2}\right)-\left(\alpha^{2}, e_{2}\right)=0, \\
\tau_{1}^{3}\left(\alpha^{2}, \alpha\right)=\left(\alpha^{3}, e_{2}\right)-\left(\alpha^{3}, e_{2}\right)=0,
\end{gathered}
$$

therefore $\tau_{1}^{3}=0$, and then $\operatorname{rank} \tau_{1}^{3}=0$.

When $n=2$, for the same reason, it follows:

$$
\begin{gathered}
\tau_{2}^{3}\left(e_{2}, \alpha^{3}\right)=\left(\alpha^{2}, \alpha\right)+\left(\alpha^{2}, \alpha\right)+\left(\alpha^{2}, \alpha\right)=3\left(\alpha^{2}, \alpha\right), \\
\tau_{2}^{3}\left(\alpha, \alpha^{3}\right)=\left(\alpha^{3}, \alpha\right)+\left(\alpha^{3}, \alpha\right)+\left(\alpha^{3}, \alpha\right)=3\left(\alpha^{3}, \alpha\right)=0, \\
\tau_{2}^{3}\left(\alpha^{2}, \alpha^{3}\right)=\left(\alpha^{4}, \alpha\right)+\left(\alpha^{4}, \alpha\right)+\left(\alpha^{4}, \alpha\right)=3\left(\alpha^{4}, \alpha\right)=0,
\end{gathered}
$$

therefore the matrix $\tau_{2}^{3}$ is 


$$
\left(\begin{array}{lll}
0 & 0 & 0 \\
0 & 0 & 0 \\
3 & 0 & 0
\end{array}\right),
$$

then $\operatorname{rank} \tau_{2}^{3}=1$.

When $n \geq 3$, and $\mathrm{n}$ is an odd number,

$$
\begin{gathered}
\tau_{n}^{3}\left(e_{2}, \alpha^{\frac{3 n-1}{2}}\right)=\left(e_{2} \alpha, \alpha^{\frac{3(n-1)}{2}}\right)-\left(\alpha e_{2}, \alpha^{\frac{3(n-1)}{2}}\right)=0, \\
\tau_{n}^{3}\left(\alpha, \alpha^{\frac{3 n-1}{2}}\right)=\left(\alpha^{2}, \alpha^{\frac{3(n-1)}{2}}\right)-\left(\alpha^{2}, \alpha^{\frac{3(n-1)}{2}}\right)=0, \\
\tau_{n}^{3}\left(\alpha^{2}, \alpha^{\frac{3 n-1}{2}}\right)=\left(\alpha^{3}, \alpha^{\frac{3(n-1)}{2}}\right)-\left(\alpha^{3}, \alpha^{\frac{3(n-1)}{2}}\right)=0 .
\end{gathered}
$$

Therefore the matrix $\tau_{n}^{3}=0$, then $\operatorname{rank} \tau_{n}^{3}=0$.

When $n \geq 4$, and $\mathrm{n}$ is an odd number,

$$
\begin{gathered}
\tau_{n}^{3}\left(e_{2}, \alpha^{\frac{3 n}{2}}\right)=\left(\alpha^{2}, \alpha^{\frac{3 n-4}{2}}\right)+\left(\alpha^{2}, \alpha^{\frac{3 n-4}{2}}\right)+\left(\alpha^{2}, \alpha^{\frac{3 n-4}{2}}\right)=3\left(\alpha^{2}, \alpha^{\frac{3 n-4}{2}}\right), \\
\tau_{n}^{3}\left(\alpha, \alpha^{\frac{3 n}{2}}\right)=\left(\alpha^{3}, \alpha^{\frac{3 n-4}{2}}\right)+\left(\alpha^{3}, \alpha^{\frac{3 n-4}{2}}\right)+\left(\alpha^{3}, \alpha^{\frac{3 n-4}{2}}\right)=3\left(\alpha^{3}, \alpha^{\frac{3 n-4}{2}}\right)=0 \\
\tau_{n}^{3}\left(\alpha, \alpha^{\frac{3 n}{2}}\right)=\left(\alpha^{4}, \alpha^{\frac{3 n-4}{2}}\right)+\left(\alpha^{4}, \alpha^{\frac{3 n-4}{2}}\right)+\left(\alpha^{4}, \alpha^{\frac{3 n-4}{2}}\right)=3\left(\alpha^{4}, \alpha^{\frac{3 n-4}{2}}\right)=0
\end{gathered}
$$

Therefore the matrix $\tau_{n}^{3}$ is

$$
\left(\begin{array}{lll}
0 & 0 & 0 \\
0 & 0 & 0 \\
3 & 0 & 0
\end{array}\right)
$$

then $\operatorname{rank} \tau_{n}^{3}=1$.

$$
\text { In conclusion, } \quad \operatorname{rank}_{n}^{3}= \begin{cases}0, & \mathrm{n} \text { is an odd number; } \\ 1, & \mathrm{n} \text { is an even number. }\end{cases}
$$

And because

$$
\operatorname{dim}_{k} H H_{n}\left(\Lambda_{3}\right)=\operatorname{dim} \operatorname{ker} \tau_{n}^{3}-\operatorname{dim} \operatorname{Im} \tau_{n+1}^{3}=\left|B_{3} \Theta A P_{3}(n)\right|-\operatorname{rank} \tau_{n}^{3}-\operatorname{rank} \tau_{n+1}^{3},
$$

Thus 


$$
\operatorname{dim}_{k} H H_{n}\left(\Lambda_{3}\right)= \begin{cases}5 & \text { when } n=0 \\ 2 & \text { when } n \geq 1\end{cases}
$$

\section{References}

[1] Hazewinkel, M. (1997). Rrpresentations of quivers and moduli of linear dynamical systems[M]. Lie Groups: History Frontiers and Application.

[2] Ibrahim, A., \& Daniel, S. (2006). Elements of the repreesentation theory of associtive algebras[M]. Techniques of Representation Theory. Cambrige Uni. Press, Carleton University.

[3] Li, L. C., \& Zhang, Y. B. (2003). Representation theory of the system quiver[J]. Science in China(Series A), 789-803.

[4] Xu, Y. G., \& Han, Y. (2007). Hochschild (co)homology of exterior algebra[J]. Comm. Alg., 35(1), 115-131.

[5] Hochschild, G. (1945). On the cohomology groups of an associatie algebra [J]. Ann.Math., 46, 58-67.

[6] Bardzell, M. J. (1997). The alternating syzygy behavior of monomial algebras[J]. J. algebra, 188, 69-89.

[7] Zhang, D. D. (2010). Hochschild cohomology groups of minimal wild system quiver algebras r[J]. Science Technology and Engineering, 8645-8648.

Chunling He was born in 1978 in Huanggang city, Hubei province, China. She graduated from Hubei University in Wuhan City, Hubei province ,with degree of master of science in 2009. Her major field is algebraic equation.

As a lecturer, she has been engaged in maths teaching since 2001 at the Huanggang Normal University. The previous publications were as follow:

He, C. L., et al. (2017). On the extremal graphs of diameter 2 with respect to the eccentric resistance-distance sum. Discrete Appl. Math., 22, 171-181. 